\title{
Proposta de Ferramenta Estatística para Controle de Estações de Tratamento de Efluentes Industriais
}

\author{
Karine Marinho do Nascimento Dias'; Gandhi Giordano' \\ karinemarinho01@gmail.com
}

1. Universidade do Estado do Rio de Janeiro - RJ .

\author{
Histórico do Artigo: \\ Recebido em: 19 de novembro de $2019 \quad$ Aceito em: 27 de abril de $2020 \quad$ Publicado em: 30 de abril de 2020
}

Resumo: Industrialmente, a água pode ser utilizada em diversos momentos, tais como a incorporação ao produto, lavagens de máquinas, tubulações, pisos, entre outros. Com a necessidade de controle dos efluentes antes do lançamento aos corpos hídricos, segundo a legislação brasileira, faz-se necessário o tratamento de efluentes industriais. Nesse sentido, as Estações de Tratamento de Efluentes Industriais (ETEIs) devem monitorar os padrões de qualidade estabelecidos por leis em relação ao efluente industrial. 0 objetivo deste trabalho foi a produção de uma ferramenta estatística capaz de analisar, através da medição indireta da matéria orgânica presente no efluente, a eficiência de uma estação de tratamento de efluentes industriais em relação à DBO e DQ0, tendo como estudo de caso um condomínio de indústrias de alimentos na cidade do Rio de Janeiro. A metodologia foi baseada na aplicação de uma ferramenta estatística utilizando o software EXCEL® ${ }^{\circledR}$ mediante os seguintes passos: imputação dos dados originais, análise descritiva exploratória, aplicação de cartas de controle, análise da eficiência da estação, produção de alerta de baixa de eficiência, apresentação dos resultados e a geração de um relatório final. Como conclusão, o resultado foi favorável em relação aos objetivos do trabalho, permitindo com que o usuário tenha, apenas em um programa, a compilação das análises indiretas da matéria orgânica e geração da eficiência da estação. Em relação ao estudo de caso, a estação apresentou eficiência acima de $97 \%$ em todos os dias analisados. Abaixo de 98\% houve quatro resultados de DB0 e um resultado de DQ0. 0 sistema identificou a data dessas análises, bem como filtrou os resultados em si, alcançando os objetivos propostos.

Palavras-chave: Cartas de controle, DBO, DQ0, Eficiência, Água.

\section{Statistical Tool Proposal for Industrial Wastewater Treatment Plants Control}

\begin{abstract}
Industrially, water can be used at different steps, such as incorporation into the product, washing machines, pipes, floors, among others. With the need to control effluents before being released to water bodies, according to Brazilian legislation, it is necessary to treat industrial effluents. In this sense, industrial wastewater treatment plants must monitor the quality standards established by laws in relation to industrial effluent, to meet the restrictions and limits imposed by the environmental license. Often the control of the station is done through several spreadsheets and reports in an individualized and non-systematized way. The objective of this work is the production of a statistical tool capable of analyzing, through the indirect measurement of organic matter present in the effluent, the efficiency of an industrial effluent treatment station in relation to BOD and COD, using a condominium case study. of food industries in the city of Rio de Janeiro. The methodology was based on the application of a statistical tool using the EXCEL ${ }^{\circledR}$ software, through the following steps: imputation of the original data, exploratory descriptive analysis, application of control charts, analysis of the station's efficiency, production of low efficiency alert, presentation results and generating a final report. As a conclusion, the result was favorable in relation to the objectives of the work, allowing the user to have, in just one program, the compilation of indirect analyzes of organic matter and generation of the station's efficiency. In relation to the case study, the station showed efficiency above $97 \%$ on all analyzed days. Below 98\% there were four BOD results and one COD result. The system identified the date of these analyzes, as well as filtered the results themselves, reaching the proposed objectives.
\end{abstract}

Keywords: Control charts, BOD, COD, Efficiency, Water. 


\title{
Análisis de la Concentración de Material Particulado (Mp2.5) en la Instalación y Operación del Corredor de Carretera Transolímpico
}

\begin{abstract}
Resumen: Industrialmente, el agua se puede utilizar en diferentes momentos, como la incorporación al producto lavadoras, tuberías, pisos, entre otros. Con la necesidad de controlar los efluentes antes de ser liberados a los cuerpos de agua, de acuerdo con la legislación brasileña, es necesario tratar los efluentes industriales. En este sentido, las estaciones de tratamiento de efluentes deben monitorear los estándares de calidad establecidos por las leyes en relación con los efluentes industriales. El objetivo de este trabajo es la producción de una herramienta estadística capaz de analizar, a través de la medición indirecta de la materia orgánica presente en el efluente, la eficiencia de una planta de tratamiento de efluentes industriales en relación con DB0 y DQ0, utilizando un estudio de caso de condomínio de industrias alimentarias en la ciudad de Río de Janeiro. La metodología se basó en la aplicación de una herramienta estadística utilizando el software EXCEL $®$, a través de los siguientes pasos: imputación de los datos originales, análisis descriptivo exploratorio, aplicación de gráficos de control, análisis de la eficiencia de la estación, producción de alertas de baja eficiencia, presentación resultados y generar un informe final. En conclusión, el resultado fue favorable en relación con los objetivos del trabajo, permitiendo al usuario tener, en un solo programa, la recopilación de análisis indirectos de materia orgánica y la generación de la eficiencia de la estación. En relación con el estudio de caso, la estación mostró una eficiencia superior al 97\% en todos los días analizados. Por debajo del 98\% hubo cuatro resultados de DB0 y un resultado de DQ0. El sistema identificó la fecha de estos análisis, así como también filtró los resultados, alcanzando los objetivos propuestos. Palabras clave: Cuadros de control, DB0, DQ0, Eficiencia, Agua.
\end{abstract}

\section{INTRODUÇ̃̃o}

A indústria de alimentos é extremamente importante no cenário econômico brasileiro. Segundo a Associação Brasileira das Indústria da Alimentação (ABIA), os grandes destaques do Brasil em 2017 foram a colocação do país como produtor e exportador mundial de suco de laranja, seguido pelo posto de segundo produtor e maior exportador mundial de carne e maior produtor e exportador de açúcar no mundo (ABIA, 2018).

A produção de alimentos coopera também para a geração de efluentes (DE RESENDE et al., 2012). Segundo os autores Giordano; Surerus (2015), efluentes líquidos industriais são o conjunto de quaisquer águas descartadas pelas indústrias.

A legislação ambiental corrobora com a realização da correta gestão ambiental (DOS SANTOS PEIXOT0, 2018). Referente a este assunto, a resolução CONAMA nº 357/2005, posteriormente alterada para CONAMA $n^{\circ} 430 / 2011$ enfatiza o correto destino dos efluentes. A resolução CONAMA n ${ }^{\circ}$ 430/2011 aponta ainda uma lista com parâmetros cujos valores não devem ter seus limites ultrapassados (BRASIL, 2011).

A demanda bioquímica de oxigênio (DB0) é definida como a quantidade de oxigênio necessária para que os micro-organismos biodegradem a matéria orgânica contida em um litro de amostra, em tempo definido e na temperatura de incubação de $20^{\circ} \mathrm{C}$. Já a DQO demanda bioquímica de oxigênio, representa a quantidade de oxigênio necessária para oxidar 
quimicamente, por via enérgica, a matéria orgânica contida em 1 litro de amostra (GIORDANO; SURERUS, 2015).

Segundo Nuvolari et al. (2003), pelo fato de oxidar os compostos orgânicos não biodegradáveis e, em certos casos, compostos inorgânicos para uma mesma amostra, o valor da DQ0 é sempre maior que o da DB0. Estes compostos interferem nos corpos d'água e na operação de sistemas biológicos de tratamento de esgoto (MILOSKI, 2015). Com relação à DQ0, os efluentes de indústrias alimentícias, exclusive pescado, com vazão maior do que $3,5 \mathrm{~m}^{3} /$ dia, devem apresentar valores menores que $400 \mathrm{mg} / \mathrm{L}$ ou $8,0 \mathrm{~kg} / \mathrm{dia}$ (INEA, 2007).

0 Controle Estatístico de Processo (CEP) é, segundo Kurokawa; Bornia (2002) uma técnica de avaliação da qualidade. Estabelecidas pelo pesquisador Dr. Walter S. Shewhart, as cartas de controle são capazes de serem utilizadas na melhoria dos processos, uma vez que a maioria deles não opera no estado classificado como “em controle estatístico” (CORRÊA; CHAVES, 2009).

0 controle dos efluentes que chegam à ETEI pode indicar falhas no processo de produção da indústria. Como exemplo, pode-se citar o trabalho de Oliveira (2007), sobre dados na área de controle de estações de tratamento, que tinha como objetivo manter a concentração média do efluente abaixo de um valor determinado a partir de análises probabilísticas (OLIVEIRA, 2007).

Para a realização deste trabalho, a autora utilizou as análises obtidas por Oliveira (2006), no qual foram avaliados cerca de 50.000 dados operacionais de 208 ETEs dos estados de Minas Gerais e São Paulo, compreendendo 21 processos de tratamentos. A avaliação teve como objetivo o desempenho do tratamento de esgoto, considerando o alcance a diversas metas de qualidade para o efluente, a influência de condições operacionais e a confiabilidade (OLIVEIRA, 2006). Já no trabalho de Wasik et al. (2018) observa-se o controle estatístico de qualidade e a melhoria dos resultados de uma estação de tratamento de águas residuais localizada nos Estados Unidos, em 2014.

Outro exemplo a ser observado foram os problemas discutidos por Rosental; Dmitruk (2014), acerca do controle de qualidade das águas residuais, visando uma estimativa de índices quantitativos, com erros em nível tolerável. Os autores utilizaram os testes estatísticos de homogeneidade dos dados. Assim, foi possível aperfeiçoar a periodicidade da coleta das amostras, dentre outros resultados positivos.

Lokhande et al. (2011) avaliaram a poluição causada por metais pesados tóxicos nos efluentes industriais no cinturão industrial Taloja de Mumbai, Índia. 0 estudo revelou que corantes e tintas oriundos de indústrias farmacêuticas e têxteis são algumas das principais causas para a poluição através de metais pesados no ambiente aquático circundante (LOKHANDE et al., 2011). 
Proposta de Ferramenta Estatística para Controle de Estações de Tratamento de Efluentes Industriais

Outros trabalhos sobre este assunto podem ser encontrados em referências tais como Sistemas de tratamento de águas residuais: modelagem, diagnóstico e controle, por Olsson e Newell (1999); Avaliação estatística multivariada de dados espaciais e variações sazonais da qualidade da água superficial, por Pejman et al. (2009), e outros como Aguado e Rosen (2008), Aguado et al. (2006), Ahmad e Reynolds (1999), Berthouex et al. (1989), Boger (1992), entre outros.

0 presente trabalho pretende, assim, produzir uma ferramenta estatística capaz de auxiliar o gerenciamento da análise indireta da matéria orgânica. Foi utilizado, dentre outros conceitos da estatística, o Controle Estatístico de Processo (CEP), através da aplicação de Cartas de Controle aos dados de uma ETEI, analisando como estudo de caso uma estação que recebe efluentes oriundos de um condomínio de fábricas de alimentos no Estado do Rio de Janeiro.

\section{MATERIAL E MÉTODOS}

A metodologia se baseou nas análises estatísticas de uma ETEI, localizada na Zona Oeste do Rio de Janeiro, que atende a um condomínio de indústrias na área de alimentos produtor de molhos, massas, sorvetes, dentre outros. Além desses, a estação também recebe o esgoto sanitário produzido nas indústrias. 0 período de análise se concentrou entre janeiro de 2012 a janeiro de 2017, período este onde os dados se mostraram em quantidade mais relevante

Os valores de DBO e DQ0 foram analisados em pontos específicos do processo. Após categorização dos dados, foi aplicada a ferramenta de Cartas de Controle, promovendo parte do Controle Estatístico de Processo do condomínio industrial estudado.

Em seguida, foi realizada a análise dos resultados, e a observação de variações ao longo do processo, associada ao histórico da ETEI, através da ferramenta estatística produzida exclusivamente para o presente trabalho. Como resultado, foi gerada uma análise sobre a situação da estação, avaliando os parâmetros identificados como importantes para a mesma. A referência utilizada para a metodologia do trabalho foi a descrição de carta de controle do livro Standard Methods for the Examination of Water and Wastewater 23th (APHA, 2012).

Como a medição indireta da matéria orgânica expressa a eficiência da estação, neste período foram monitorados os valores de DBO e DQ0 em pontos específicos do processo, sendo estes Saída Aeróbio, Efluente Tratado, Efluente Bruto, Saída do tanque Primário, Saída do tanque Anaeróbio e Tanque de Aeração. Os parâmetros DBO e DQO possuem relação com a matéria orgânica presente no efluente. Sendo assim, a análise desses parâmetros pode ser considerada como principal para a classificação de uma ETEI. 
Foi considerado tecnicamente aceitável pelo gestor responsável da ETEI de alimentos que, valores acima de $98 \%$ de eficiência seriam aceitáveis para a operação da estação. A eficiência encontrada abaixo de $98 \%$ será então um dos pontos de interesse da ferramenta, objetivando identificar quais dias esses valores seriam encontrados, uma vez que, identificando os dias de eficiência abaixo de 98\%, o gestor da estação pode fazer uma busca nos relatos do dia, procurando obter informações sobre aquele efluente especificamente.

A ferramenta estatística foi nomeada como "ETEI Control'. Foi produzida em software Microsoft EXCEL@ 865 , de acordo com as etapas a seguir:
a) entrada dos dados;
b) análise descritiva exploratória;
c) cartas de controle;
d) eficiência da estação;
e) alerta de baixa de eficiência;
f) apresentação dos resultados;
g) geração de um relatório final.

\section{RESULTADOS E DISCUSSÃ0}

Inicialmente, a primeira aba da ferramenta, intitulada de "Intro", apresenta a descrição da estação e informações básicas para serem inseridas pelo usuário, como Código da Estação, Endereço, Cidade, Responsável, Data da Operação, Operador, Observações, como visto na Tabela 1.

Tabela 1. Aba "Intro"

\begin{tabular}{|ll|}
\hline \multicolumn{2}{|c|}{ Controle Automático- Estação de Tratamento de Efluente Industrial } \\
\hline Nome da Estação: & ESTAÇÃO MARACANÃ \\
\hline Endereço: & AVENIDA DAS ESTAÇõES, MARACANÃ, \\
\hline Cidade: & Rio de Janeiro \\
\hline Responsável: & ENGENHEIRO JOSÉ DA SILVA \\
\hline Data da operação: & $20 / 12 / 2017$ \\
\hline Operador: & TÉCNICO ANTŌNIO DAS NEVES \\
\hline Observaçōes: & PROCURO SABER A EFICIÊNCIA DA ESTAÇÃO \\
\hline
\end{tabular}

Fonte: A autora, 2019.

A segunda e terceira abas, intituladas “In_DB0" e "In_DQ0" respectivamente, são as abas reservadas para o usuário inserir os dados referentes à DBO e DQO da estação. Como pode ser 
Proposta de Ferramenta Estatística para Controle de Estações de Tratamento de Efluentes Industriais

visto na Tabela 2, exemplo para DB0, as células devem constar as datas no formato DD/MM/AAAA - data, mês e ano- os dados de DB0 e DQ0 em cada ponto de aferição indicado na legenda. Em caso de valores faltantes, é imprescindível que a célula da planilha esteja em branco.

Tabela 2. Inserção de dados de DBO

\begin{tabular}{|c|c|c|c|c|c|c|}
\hline DATA & EFL_BRUTO & SAIDA_DO_PRIMARIO & SAIDA_ANAEROBIO & SAIDA_AEROBIO & EFL_TRATADO & VAZÃo \\
\hline $03 / 01 / 2012$ & 1146 & 637 & 558 & 13 & 17 & 100 \\
\hline $31 / 01 / 2012$ & 1978 & 733 & 765 & 13 & 16 & 70 . \\
\hline $10 / 02 / 2012$ & 1804 & 1260 & 759 & 5 & 10 & $50^{\circ}$ \\
\hline 07/03/2012 & 2424 & 1262 & 3 & 54 & 5 & 60 \\
\hline $03 / 04 / 2012$ & 7734 & 1941 & 302 & 5 & 14 & 20 \\
\hline $02 / 05 / 2012$ & 3669 & 754 & 304 & 9 & 21 & 60 \\
\hline $05 / 06 / 2012$ & 1694 & 550 & 107 & 5 & 9 & 50 \\
\hline $03 / 07 / 2012$ & 2715 & 785 & 307 & 4 & 3 & 40 \\
\hline $01 / 08 / 2012$ & 2415 & 585 & 260 & 4 & 5 & 50 \\
\hline $04 / 09 / 2012$ & 2130 & 300 & 241 & 3 & 3 & 40 \\
\hline $09 / 10 / 2012$ & 1956 & 400 & 246 & 3 & 3 & 60 \\
\hline $12 / 11 / 2012$ & 1392 & 299 & 185 & 3 & 3 & 70 \\
\hline $05 / 12 / 2012$ & 1740 & 599 & 426 & 3 & 3 & 70 , \\
\hline
\end{tabular}

A quarta aba, denominada "Descritiva_Tabela” é referente as análises descritivas sobre o conjunto de observações com as principais medidas de dispersão. As medidas de dispersão são parâmetros estatísticos usados para determinar o grau de variabilidade dos dados de um conjunto de valores e foram analisadas de acordo com os seguintes indicadores: quantidade válida, valor mínimo, média, mediana, máximo, desvio padrão, coeficiente de variação, percentis e quartis de todas as variáveis $(90 \%, 75 \%, 25 \%, 10 \%)$ e média anual dos anos utilizados (2012 a 2017).

A quinta aba, denominada “Descritiva_Gráfico”, apresenta a série histórica dos conjuntos de dados de DBO e DQ0 separados por variáveis. A sexta aba, denominada "Carta_de_Controle" apresenta duas áreas de visualização. A primeira é referente aos gráficos de carta de controle, com os respectivos limites aplicados, indicado como parte 01 da aba "Carta_de_Controle", como observado no Gráfico 1. 
Gráfico 1. Aba “Carta_de_Controle” parte 01

\begin{tabular}{c}
\hline $\bar{E} \equiv$ \\
ETEIControl \\
\hline
\end{tabular}

\section{Cartas de Controle}



Fonte: A autora, 2019. 
Proposta de Ferramenta Estatística para Controle de Estações de Tratamento de Efluentes Industriais

Já a parte 02 é referente as variáveis auxiliares de DBO e DQ0, que são as cópias das informações iniciais das abas dois e três. Acrescidas a essas, também são apresentadas as colunas com as variáveis de limites superior e inferior, através de suas respectivas fórmulas.

Dentro dos cálculos das cartas de controle estão o limite central, intitulado LC, e os limites superiores de controle, a seguir: (a) LSC 1: primeiro limite superior de controle; (b) LSC2: segundo limite superior de controle; (c) LSC3: terceiro limite superior de controle.

Os limites inferiores de controle também foram abordados, a seguir: (a) LIC 1: primeiro limite inferior de controle (b) LC2: segundo limite inferior de controle (c) LC3: terceiro limite inferior de controle. Todos os pontos de coleta foram identificados pelas respectivas variáveis. Assim, cada ponto de coleta possui uma tabela com os cálculos produzidos e o gráfico gerado.

Como pode ser visto na Tabela 3, a aba denominada "Eficiência" apresenta as análises descritivas sobre o conjunto de observações com as principais medidas de dispersão, centralidade e cartas de controle.

Caso o valor esteja abaixo de 98\% de eficiência, o sistema identifica este valor com uma coloração vermelha. Este valor de eficiência pode ser alterado pelo usuário e a planilha identificará automaticamente as novas eficiências em coloração vermelha.

Tabela 3. Cálculo de eficiência, com indicador de baixa eficiência.

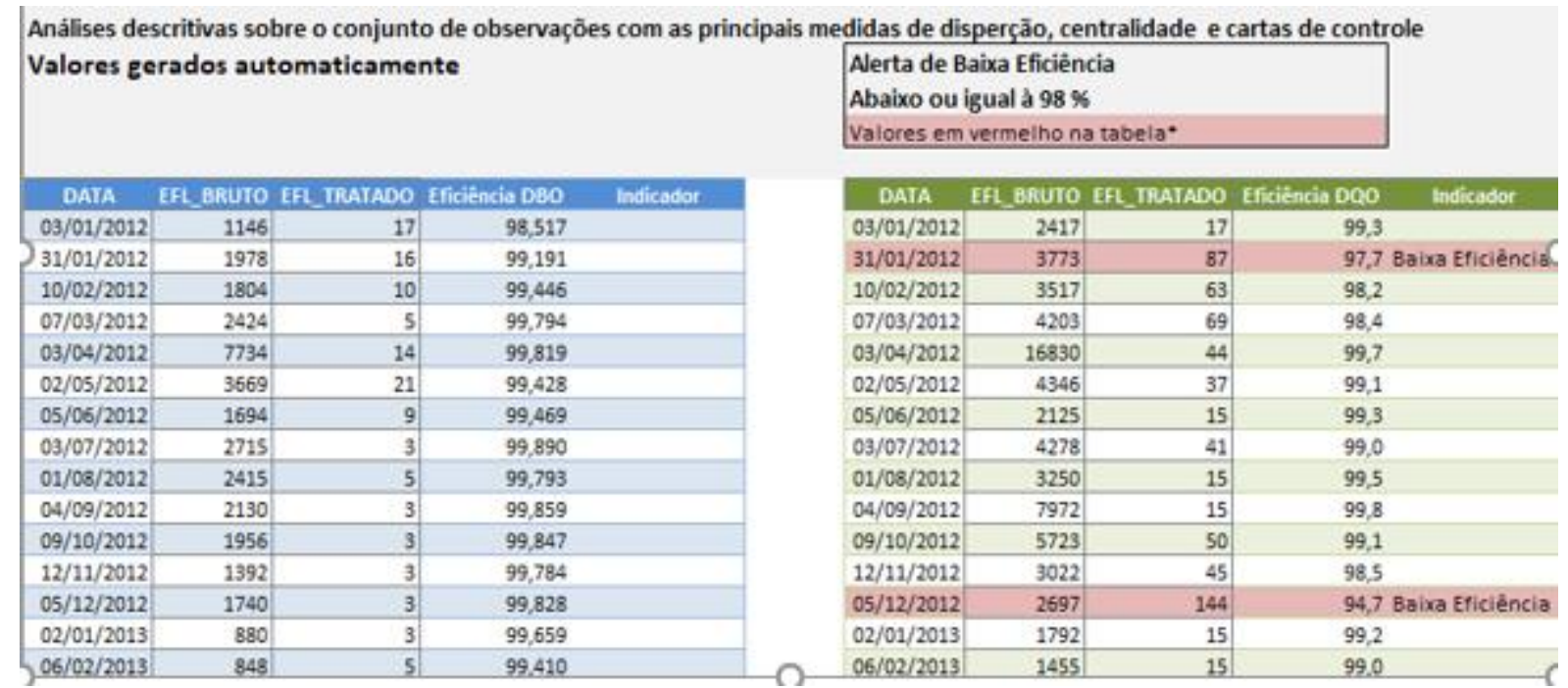

Fonte: A autora, 2019 
Também são gerados os gráficos de eficiência, tanto da DBO quanto da DQO. Na área entre os gráficos de eficiência está inserida a seta denominada "Relatórios", onde permite ao usuário seguir automaticamente para a próxima aba do sistema.

A aba seguinte, denominada "Alerta de baixa eficiência", resume todos os principais resultados descritos na aba anterior, denominada “Eficiência”. Dessa forma, caso um dos valores seja indicado como baixa eficiência, automaticamente as informações sobre data, efluente bruto, efluente tratado e eficiência da DB0 aparecerão nesta tabela, resumindo para o usuário quais datas e informações ele deve checar ao procurar solucionar o problema.

Na referida aba foi utilizado o recurso "macro", do EXCEL®, possibilitando o retorno à página anterior. Já a aba "Resultados" apresenta os resultados analíticos do processo, como observado no Gráfico 2. A primeira parte dos resultados é a eficiência da estação, que tem sua demonstração a partir da escolha do ano de análise, feita pelo usuário. Em sequência, são gerados os gráficos de eficiência do ano em questão, expostos em porcentagem.

Gráfico 2. Aba "Resultados" - gráficos de eficiência da DB0 e DQ0

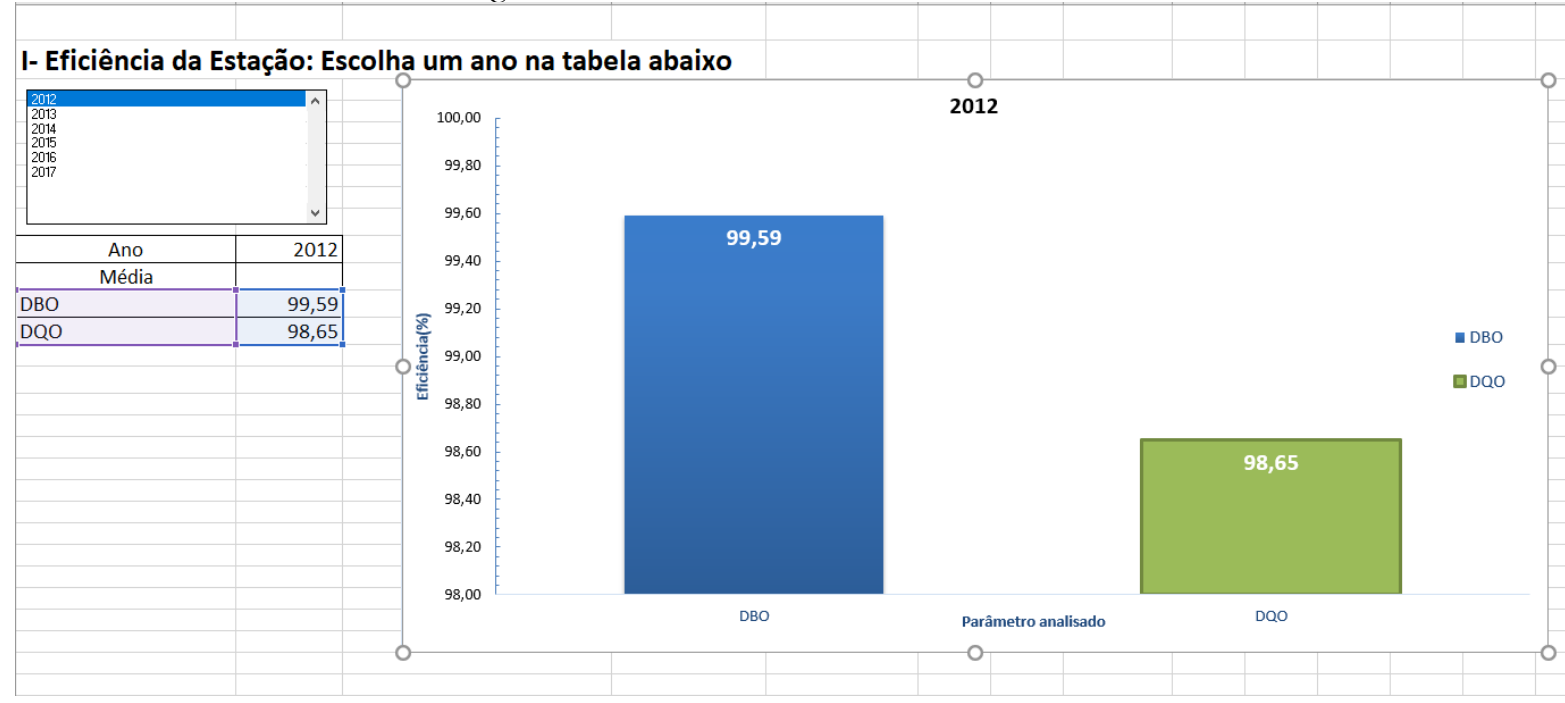

Fonte: A autora, 2019.

A segunda parte refere-se aos valores de média anual do efluente, que indicam as médias anuais de DBO e DQ0, em cada ano de estudo. Concluindo a tabela, tem-se a última aba denominada “Relatório”. Nesta, o operador pode gerar um relatório resumido em tabela EXCEL® sobre a eficiência da estação, contabilizando os resultados encontrados a partir dos padrões estabelecidos pelo operador do sistema, conforme Figura 1. 


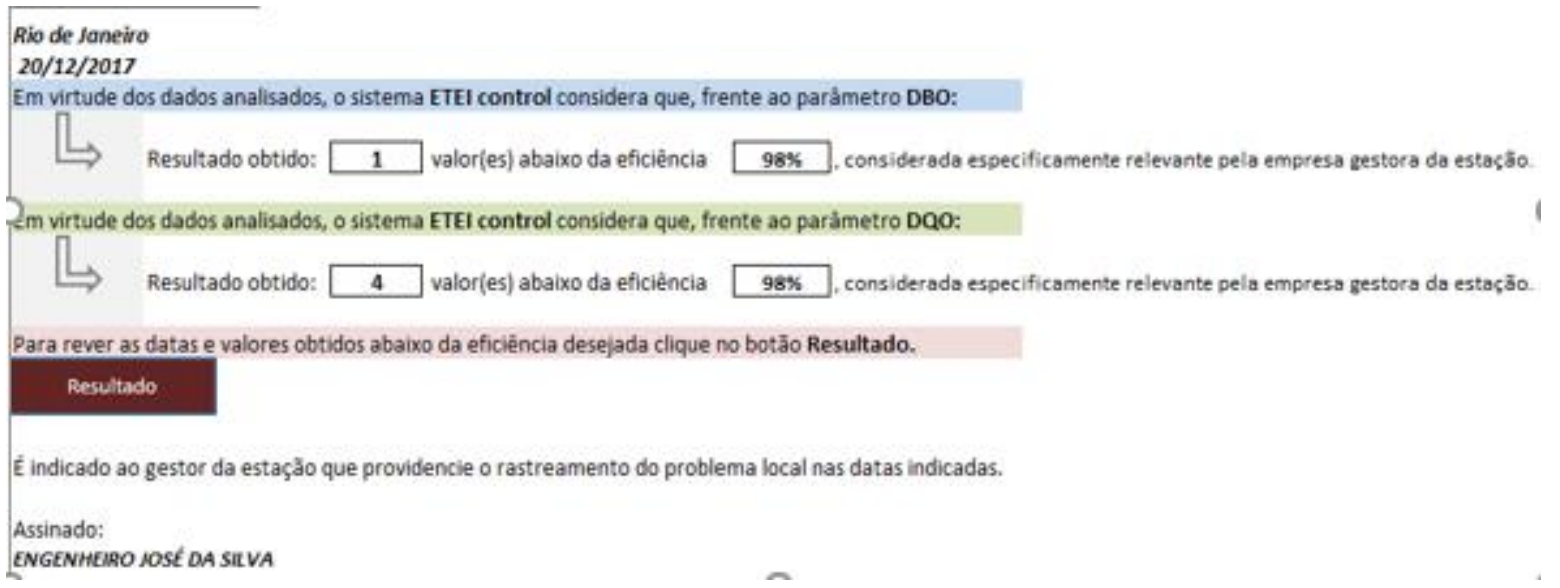

Figura 1. Relatório final do sistema ETEI Control Fonte: A autora, 2019.

A partir da produção da ferramenta, foi realizada a avaliação da ETEI segundo o sistema ETEI Control. A seguir estão descritos os resultados da produção da ferramenta estatística bem como da situação da ETEI em estudo. Através do presente trabalho foi possível gerar uma ferramenta, em software Microsoft EXCEL $\circledast$, capaz de analisar automaticamente a eficiência de uma estação de tratamento de efluentes, observando os valores de DB0 e DQ0.

Seguindo os objetivos estipulados, foi possível produzir, através da análise descritiva e exploratória dos dados gráficos, relatórios para uma melhor compreensão dos fenômenos existentes.

Também foi analisado o comportamento da série histórica das amostras, através da ferramenta estatística produzida. Neste ponto, foram produzidas as cartas de controle, com 0 objetivo de verificar a ocorrência de falhas e outros eventos raros nos processos. Por fim, foram analisados os resultados obtidos nas etapas anteriores.

A ferramenta foi produzida com sucesso, identificando os valores de eficiência abaixo de 98\%, assim como as datas em que esses valores ocorreram.

A partir das análises descritivas, é possível observar os resultados comparativos das médias dos valores de DBO e DQ0 de todos os anos, como segue nos Gráficos 3 e 4.

Gráfico 3. Valores da Média DB0 


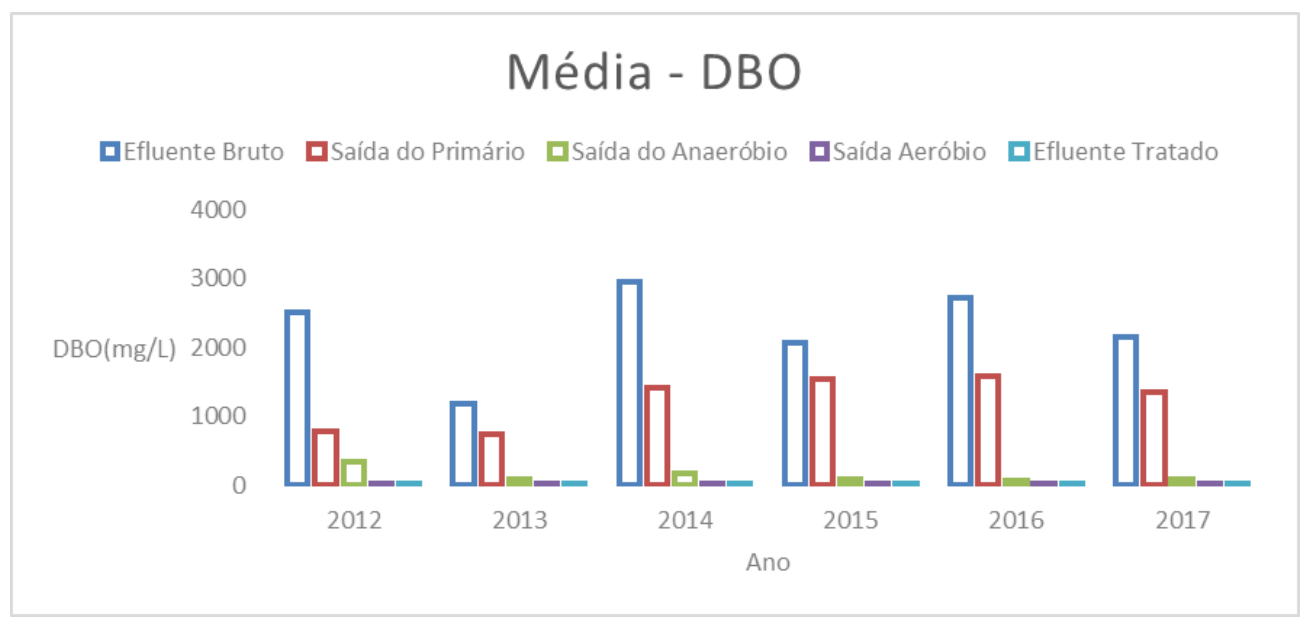

Fonte: A autora, 2019.

É possível observar que, apesar de todos os anos o valor de efluente bruto analisado foi acima de $2000 \mathrm{mg} / \mathrm{L}$, o tratamento possibilitou grande redução nos níveis de DB0, possibilitando resultados altamente expressivos.

Gráfico 4. Valores da Média- DQ0

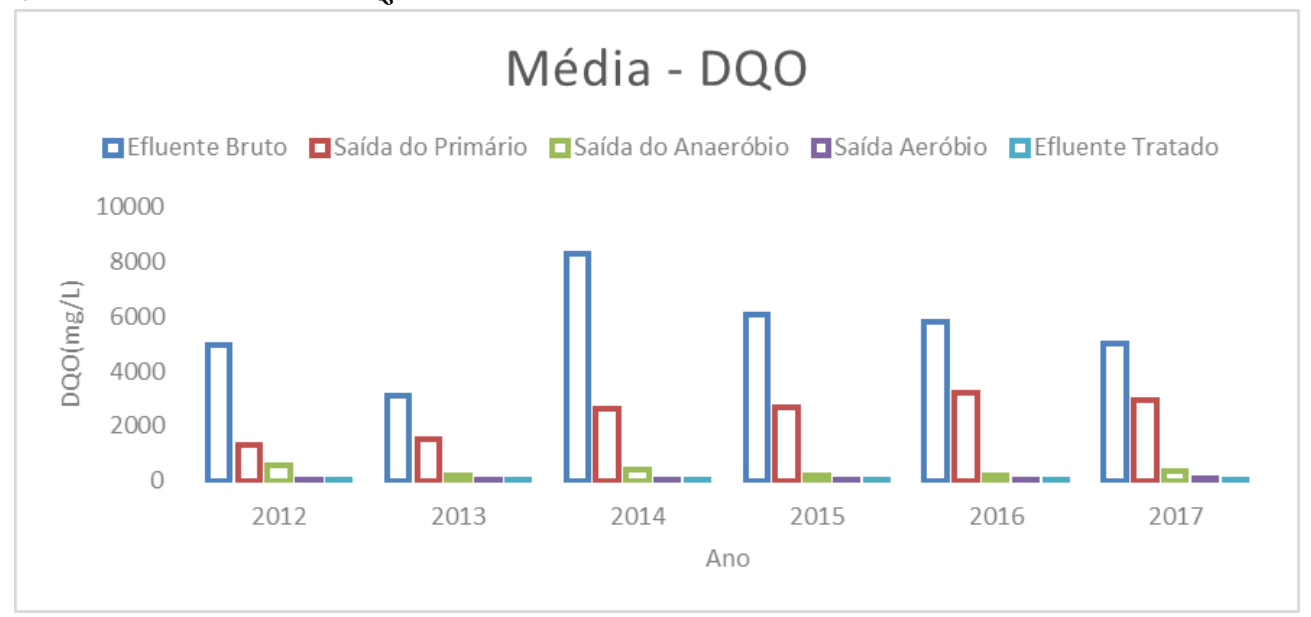

Fonte: A autora,2019.

Neste caso pode-se indicar a eficiência no tratamento pela diferença de escala entre 0 efluente bruto e o efluente tratado. Entretanto, para melhor avaliação do efluente tratado, observa-se nos Gráficos 5 e 6 a real situação do efluente, tanto para DB0 quanto DQ0. 
Proposta de Ferramenta Estatística para Controle de Estações de Tratamento de Efluentes Industriais

Gráfico 5. Valor de efluente tratado - DB0

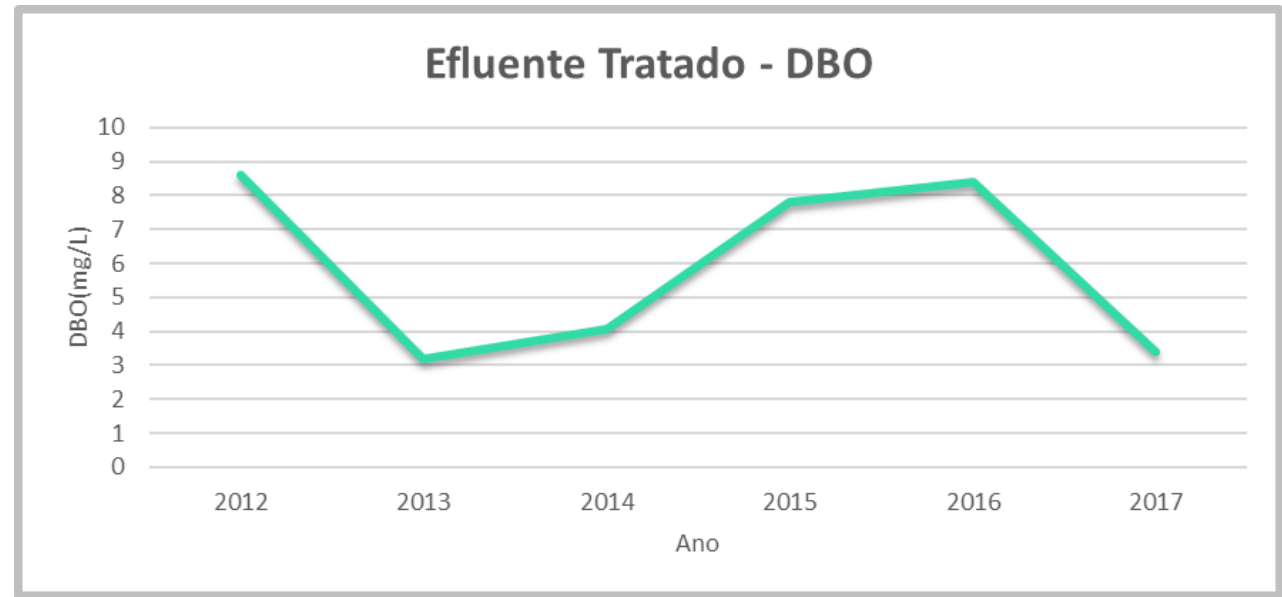

Fonte: A autora, 2019.

Em ambos os casos, o efluente tratado demonstra a eficácia do tratamento. Em relação à DB0, os valores se mantiveram abaixo de $10 \mathrm{mg} / \mathrm{L}$, como observado no Gráfico 5 . Já para valores de DQ0, eles se mantiveram abaixo de $60 \mathrm{mg} / \mathrm{L}$, como observado no Gráfico 6.

Gráfico 6. Valor de efluente tratado - DQO

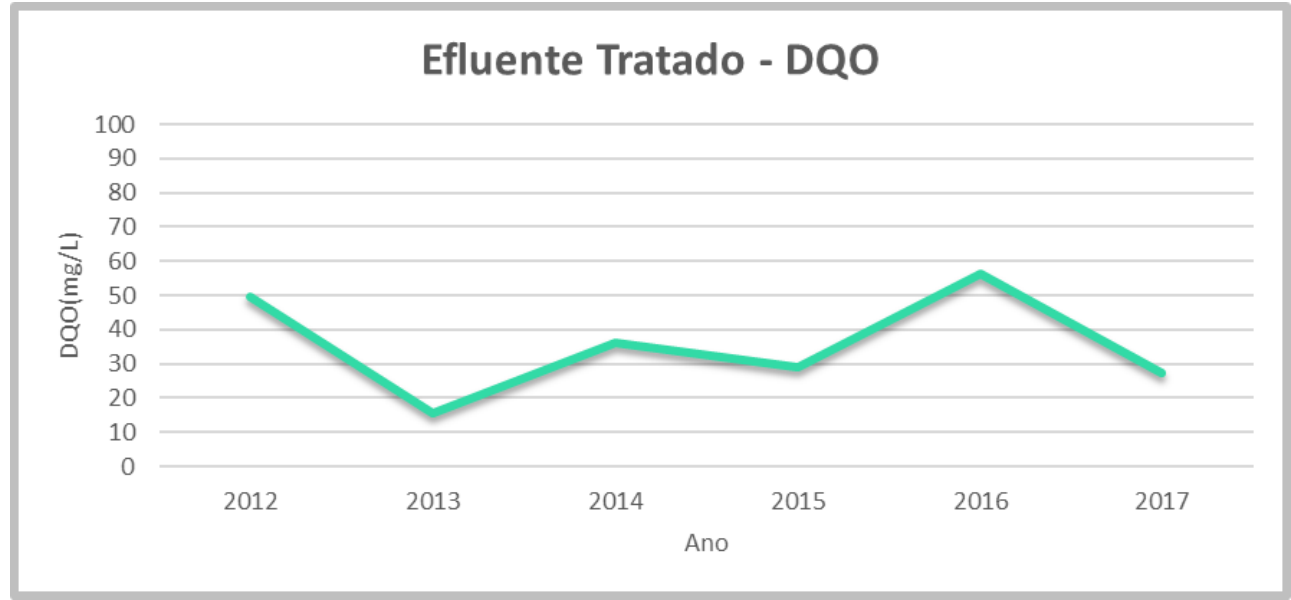

Fonte: A autora, 2019.

As cartas de controle foram também aplicadas a todos os pontos de coleta. Com o objetivo de analisar a resposta final do processo, seguem os Gráficos 7 e 8, que demonstram as cartas de controle do efluente tratado. 
Gráfico 7. Carta de controle - Efluente tratado DBO

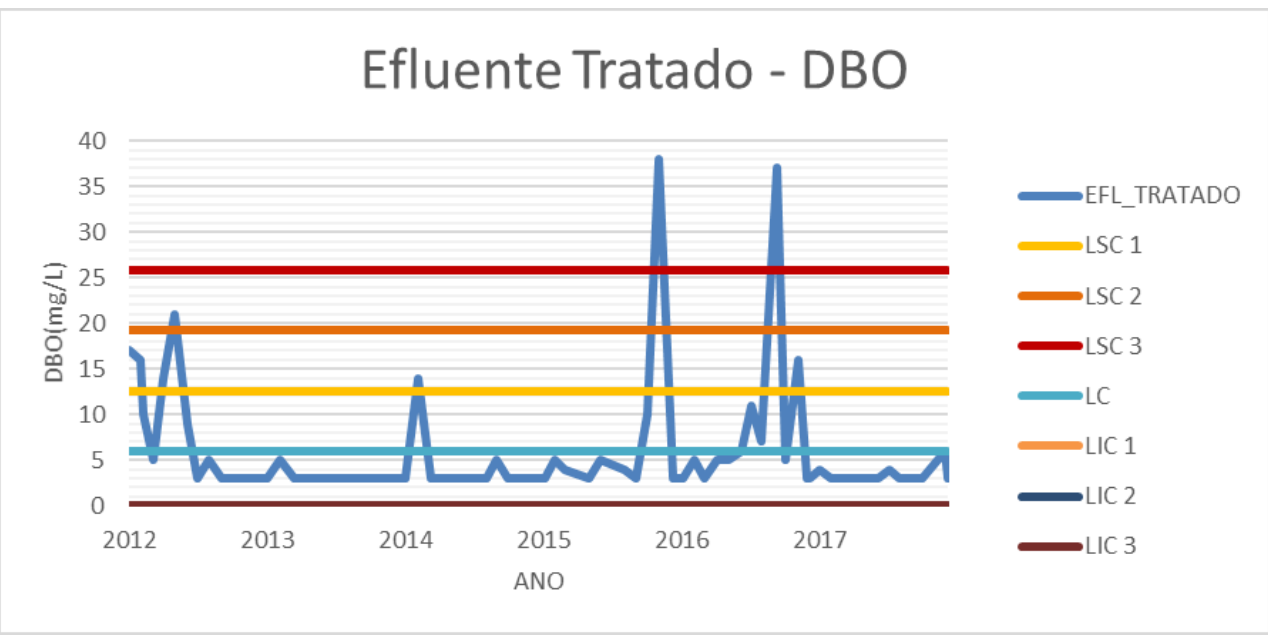

Legenda: LSC 1: Primeiro limite superior de controle; LSC 2: Segundo limite superior; LSC 3: Terceiro limite superior; LC: Limite central; LIC 1: Primeiro limite inferior; LIC 2: Segundo limite inferior; LIC 2: terceiro limite inferior. Fonte: A autora, 2019.

No gráfico 7 é possível analisar que o valor do terceiro limite de controle foi ultrapassado apenas duas vezes em todo histórico da análise.

Gráfico 8. Carta de controle - Efluente tratado DQ0

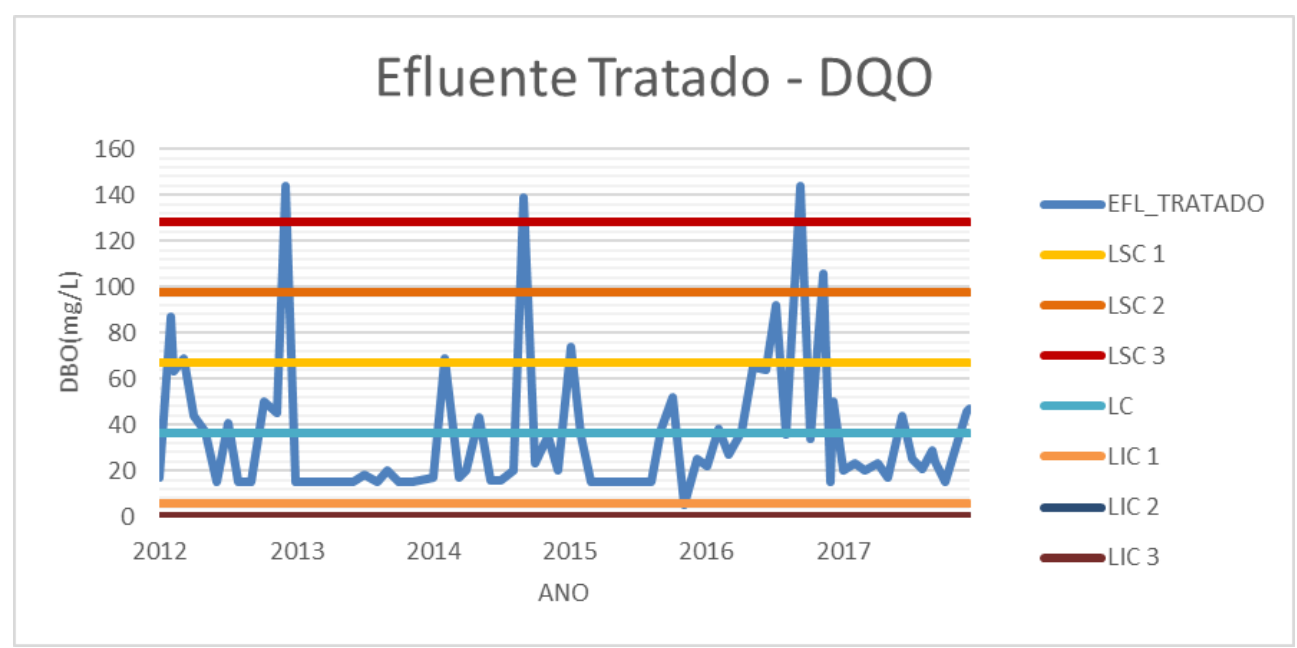

Legenda: LSC 1: Primeiro limite superior de controle; LSC 2: Segundo limite superior; LSC 3: Terceiro limite superior; LC: Limite central; LIC 1: Primeiro limite inferior; LIC 2: Segundo limite inferior; LIC 2: terceiro limite inferior. Fonte: A autora, 2019.

Já o gráfico de DQ0 teve o terceiro limite de controle ultrapassado três vezes, ao longo dos cinco anos de observações. De acordo com os gráficos acima apresentados, o cálculo da eficiência vem corroborar os resultados obtidos. A seguir estão os Gráficos 9 e 10 de eficiência de DB0 e DQ0 da estação em estudo. 
Proposta de Ferramenta Estatística para Controle de Estações de Tratamento de Efluentes Industriais

Gráfico 9. Eficiência da DB0

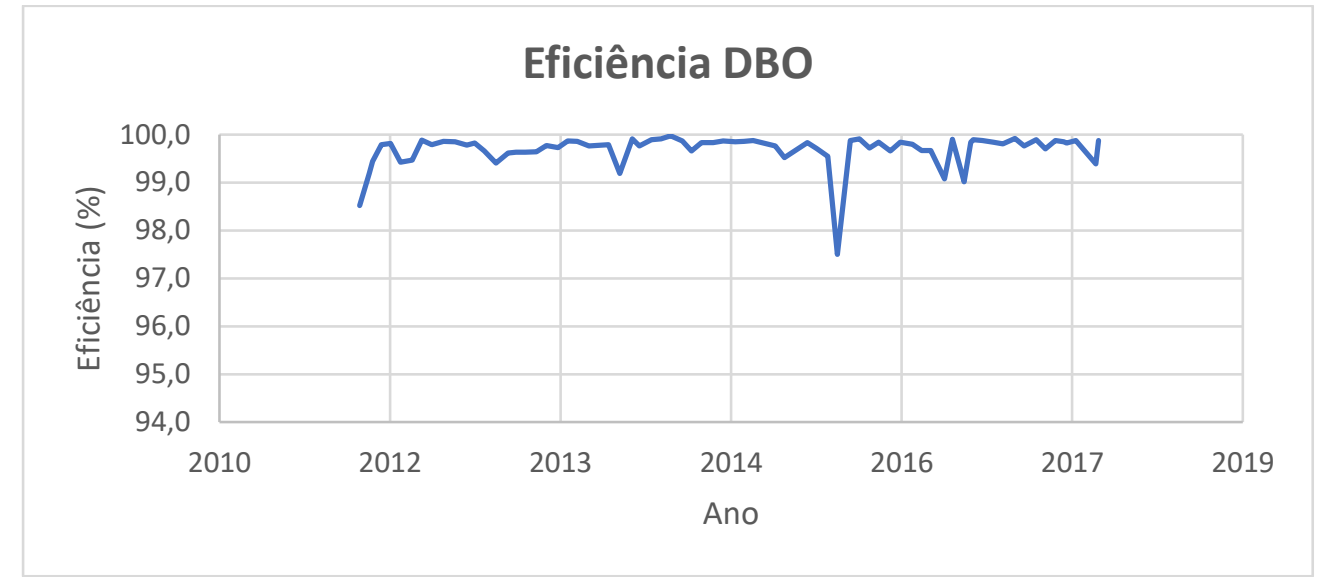

Fonte: A autora, 2019.

Em ambas as análises de eficiência, os valores se mantiveram acima dos 90\%. Entretanto, é possível observar através do Gráfico 9 que a eficiência mínima de DB0 está em torno de 97\%, enquanto a eficiência mínima de DQ0 está em 94\%, de acordo com o Gráfico 10, visto a seguir.

Gráfico 10. Eficiência da DQQ0

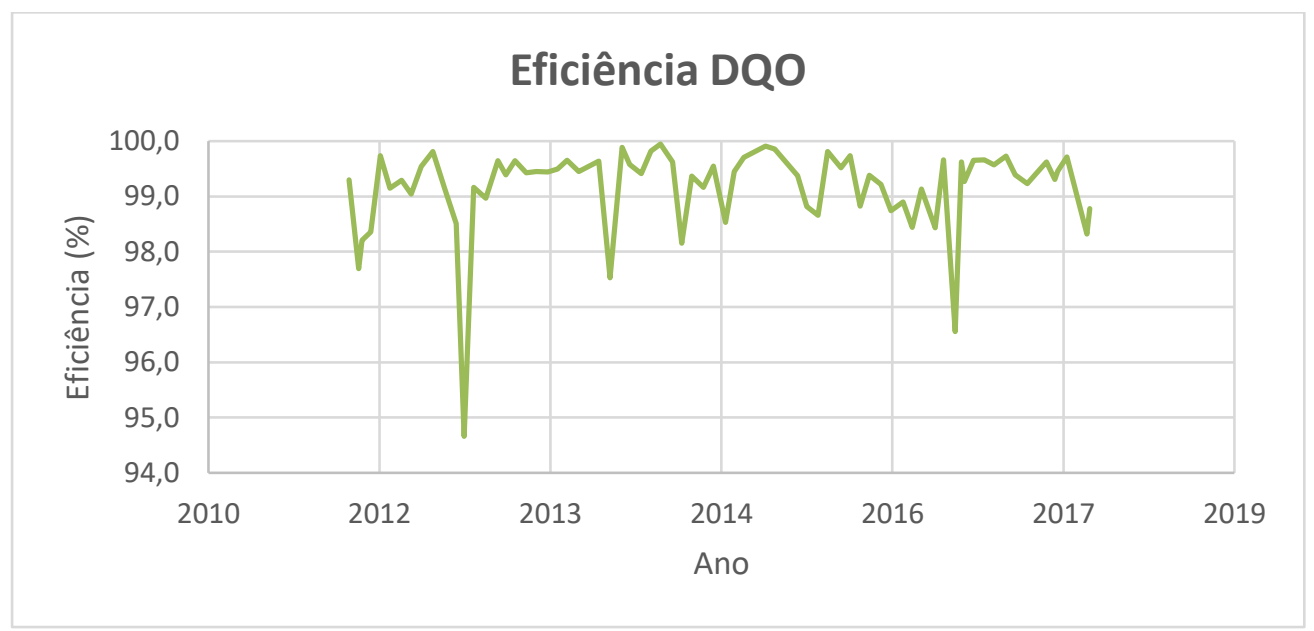

Fonte: A autora, 2019.

De acordo com o resultado do sistema ETEI Control, a estação de tratamento analisada apresentou eficiência alta em todos os anos, tanto nos processos de DB0 quanto DQ0. Os valores se mantiveram acima de 94\% em toda a série histórica analisada.

Sendo o objetivo de o usuário estipular uma pesquisa que identificasse todas as eficiências abaixo de 98\%, valor este considerado pela gestora da unidade como sendo apropriado para entrega aos clientes, o sistema através da aba "Relatório" possibilitou a 
identificação rápida dos valores abaixo de 98\%, e assim foi possível a observação das datas em que ocorreram essas eficiências.

Dessa forma, o usuário é capaz de produzir uma pesquisa localizada, nas datas indicadas pelo sistema ETEI Control, de forma que as respostas para a situação encontrada nos dias apresentados pelo sistema sejam dadas de maneira mais rápida.

É possível observar que a eficiência de DB0 apresentou valor abaixo do esperado somente na data de 03/11/2015, sendo o valor do Efluente bruto de $1502 \mathrm{mg} / \mathrm{L}$ e o efluente tratado de 38mg/L, gerando uma eficiência de 97\%. Já o resultado de DQ0 apresentou eficiência abaixo do esperado em mais dias, como é visto na Tabela 4.

Tabela 4. Eficiência de DQ0 abaixo do esperado.

\begin{tabular}{cccc}
\hline Data & Efl_ Bruto $(\mathrm{mg} / \mathrm{L})$ & Efl_Tratado $(\mathrm{mg} / \mathrm{L})$ & Eficiência \\
\hline $31 / 01 / 2012$ & 3773 & 87 & 97,7 \\
$05 / 12 / 2012$ & 2697 & 144 & 94,7 \\
$04 / 02 / 2014$ & 2794 & 69 & 97,5 \\
$09 / 11 / 2016$ & 3076 & 106 & 96,6 \\
\hline
\end{tabular}

Fonte: A autora, 2019.

Dessa maneira, considera-se que a ETEI analisada tem seus padrões de eficiência em excelente situação e entrega aos clientes uma condição altamente favorável de qualidade da estação. Os demais gráficos gerados pela ferramenta encontram-se nos anexos deste trabalho.

\section{CONCLUSÃ0}

Sobre a produção da ferramenta, conclui-se que foi apresentado um resultado favorável em relação ao seu objetivo proposto. As análises realizadas pelo sistema ETEI Control podem ser feitas para outras estações de tratamento, com as devidas alterações nos dados e indicações de referência em eficiência da qual o usuário deseje observar.

Além disso, sobre a ferramenta estatística produzida é possível incorporar outros parâmetros físico-químicos presentes na rotina de análise da ETEI, porém não contemplados nesse trabalho. Da mesma forma, é possível incorporar outras funções estatísticas, de acordo com a necessidade de cada estação a ser observada. 
Em trabalhos futuros, é sugerido que se faça a validação da ferramenta também em outras estações de tratamento. Isso porque diferentes situações podem ser encontradas e a equalização dessas situações através da ferramenta trará um aumento na qualidade da tabela.

Outra sugestão é a codificação do produto criado através do software Microsoft EXCEL® para linguagens de programação, no qual ele poderá ser efetivamente utilizado como uma API (interface de programação de aplicações), integrando as informações e resultados obtidos a sistemas maiores.

Sobre as observações estatísticas feitas através dos dados da ETEI analisada, comparando com a legislação C0NAMA 430/2011, é possível afirmar que, considerando os valores de DB0 e DQ0, a estação de tratamento de efluentes avaliada neste trabalho atende aos padrões observados pela legislação, com eficiência acima de 94\%, no intervalo de avaliação dos dados (anos de 2012 à 2017). Os valores encontrados abaixo de 98\% foram identificados, auxiliando o gestor da unidade na busca pelo fator que alterou a eficiência da estação, sendo este interno ou externo à ETEI, alcançando o objetivo do trabalho.

\section{REFERÊNCIAS BIBLIOGRÁFICAS}

ABIA (Brasil). Indústria da Alimentação em 2017. 2018. Disponível em: <https://www.abia.org.br/vsn/temp/z2018412NumerosdoSetor2017oti.pdf>. Acesso em: 26 jul. 2018.

AGUADO, D. et al. Comparison of different predictive models for nutrient estimation in a sequencing batch reactor for wastewater treatment. Chemometrics and intelligent laboratory systems, 2006.

AGUADO, D.; ROSEN, C. Multivariate statistical monitoring of continuous wastewater treatment plants. Engineering Applications of Artificial Intelligence, v. 21, n. 7, p. 1080-1091, 2008.

AHMAD, S. R.; REYNOLDS, D. M. Monitoring of water quality using fluorescence technique: prospect of on-line process control. Water Research, 1999.

APHA, AWWA, WEF. Standard Methods for examination of water and wastewater. 22nd ed. Washington: American Public Health Association; 2012.

BERTHOUEX, P. M.; LAI, W.; DARJATMOK0, A. Statistics-based approach to wastewater treatment plant operations. Journal of Environmental Engineering, 1989.

BOGER, Z. Application of neural networks to water and wastewater treatment plant operation. ISA transactions, 1992.

BRASIL. Resolução CONAMA nº 357, de 17 de março de 2005. Ministério do Meio Ambiente. Classificação de águas, doces, salobras e salinas do Território Nacional (in Portuguese), 2005.

BRASIL. Resolução CONAMA n⿳0 430, de 13 de maio de 2011. Dispõe sobre as condições e padrões de lançamento de efluentes, complementa e altera a Resolução no 357, de 17 de março de 2005, do Conselho Nacional do Meio Ambiente-CONAMA. Diário Oficial da União, 2011.

CORREAA, J. M.; CHAVES, A. N. Estudo do controle e análise da capacidade do processo de produção de água potável. XLI SBPO, 2009. 
DE RESENDE, D. S.; DA SILVA BEZERRA, A. C.; DE GOUVEIA, A. M. C. Propriedades mecânicas de compósitos cimentícios produzidos com lodo de estação de tratamento de efluentes da indústria de batata pré-fritas. Rem: Revista Escola de Minas, 2012.

DOS SANTOS PEIXOTO, D. R. A importância da legislação ambiental para a gestão ambiental pública municipal e no setor privado. Revista Internacional de Ciências, 2018.

GIORDANO, G.; SURERUS, V. Efluentes industriais, volume 1: estudo de tratabilidade volume 1.l ed. Rio de Janeiro: Publit, 2015.

INEA (INSTITUTO ESTADUAL D0 AMBIENTE) - Diretriz 205 R.06. Diretriz de Controle de Carga Orgânica em Efluentes Líquidos de origem Industrial. Rio de Janeiro, 2007.

KUROKAWA, E.; BORNIA, A. C. Uma proposta para a utilização do controle estatístico do processo (CEP) através da carta "X" como uma ferramenta gerencial para a avaliação da vazão mínima noturna de um setor. Planejamento, projeto e operação de redes de abastecimento de água, 2002.

LOKHANDE, R. S.; SINGARE, P. U.; PIMPLE, Deepali S. Toxicity study of heavy metals pollutants in waste water effluent samples collected from Taloja industrial estate of Mumbai, India. Resources and Environment, 2011.

MILOSKI, J. Caracterização de microbiota envolvida nos processos aeróbios (lodos ativados) e anaeróbios (UASB) de uma indústria de alimentos. 2015. Dissertação (Mestrado em Engenharia Ambiental) - Faculdade de Engenharia, Universidade do Estado do Rio de Janeiro, Rio de Janeiro.

NUVOLARI, A. et al. Esgoto sanitário: coleta, transporte, tratamento e reuso agrícola. 2003.

OLIVEIRA, S. C.; VON SPERLING, M. Análise da confiabilidade de estações de tratamento de esgotos. Engenharia Sanitária e Ambiental, 2007.

OLIVEIRA, S. M. A. C. Análise de desempenho e confiabilidade de estações de tratamento de esgotos. Tese (Doutorado em Saneamento, Meio Ambiente e Recursos Hídricos) - Escola de Engenharia, Universidade Federal de Minas Gerais, Belo Horizonte, 231 f. 2006.

OLSSON, G.; NEWELL, B. Wastewater treatment systems. IWA publishing, 1999.

PEJMAN, A. H. et al. Evaluation of spatial and seasonal variations in surface water quality using multivariate statistical techniques. International Journal of Environmental Science \& Technology, 2009.

ROSENTAL, 0.; DMITRUK, V. Corpopate quality measurement for analysis of stability of industrial water use and reliability of water control. Journal of Water Chemistry \& Technology, 2014.

WASIK, E. et al. Stability Monitoring of the Nitrification Process: Multivariate Statistical Analysis. Polish Journal of Environmental Studies, 2018. 